\title{
PCR-mediated repeated chromosome splitting in Saccharomyces cerevisiae
}

\author{
Minetaka Sugiyama, Shigehito Ikushima, Toshimasa Nakazawa, \\ Yoshinobu Kaneko, and Satoshi Harashima
}

BioTechniques 38:909-914 (June 2005)

\begin{abstract}
Chromosome engineering is playing an increasingly important role in the functional analysis of genomes. A simple and efficient technology for manipulating large chromosomal segments is key to advancing these analyses. Here we describe a simple but innovative method to split chromosomes in Saccharomyces cerevisiae, which we call PCR-mediated chromosome splitting (PCS). The PCS method combines a streamlined procedure (two-step PCR and one transformation per splitting event) with the Cre/loxP system for marker rescue. Using this novel method, chromosomes I $(230 \mathrm{~kb})$ and XV (1091 kb) of a haploid cell were split collectively into 10 minichromosomes ranging in size from $29-631 \mathrm{~kb}$ with high efficiency (routinely 80\%) that were occasionally lost during mitotic growth in various combinations. These observations indicate that the PCS method provides an efficient tool to engineer the yeast genome and may offer a possible approach to identify minimal genome constitutions as a function of culture conditions through further splitting, followed by combinatorial loss of minichromosomes.
\end{abstract}

\section{INTRODUCTION}

Genome engineering technology has made a significant contribution to understanding the functional and organizational characteristics of the genome from prokaryote to eukaryote (1-5). Resolving a huge chromosome into its constituent parts further facilitates structural and functional dissection of a complex genome. For example, separation of a specific chromosomal region from the intact chromosome may lead to the functional identification of the particular region having an autonomously replicating sequence (ARS). In previous studies, we developed a novel chromosome engineering technique in haploid Saccharomyces cerevisiae cells, which we called chromosome splitting (Figure 1; References 6 and 7). Although the chromosome-splitting technique is an extension of chromosomefragmenting techniques (8), the former generates two smaller chromosomes whereas the latter deletes one side of a chromosome. Chromosome splitting can also be applied to replace, fuse, or transfer chromosome segments $(9,10)$. However, the conventional technique is still inefficient, complex, and timeconsuming due to the need to clone the target sequence into a splitting vector. Moreover, the availability of selectable markers restricts repeated splitting experiments at multiple chromosomal sites. These obstacles seriously hamper a wider application of this technology.

Thus, in the current study, we have further developed a simple chromosomesplitting method. The significant new features of this method are (i) the preparation of splitting fragments by PCR and (ii) the use of recyclable markers. These improvements greatly facilitate the procedure for repeated chromosome splitting and increase the reliability of this technology for a variety of applications in haploid yeast cells. The feasibility of this method was investigated by repeated splitting of chromosomes I $(230 \mathrm{~kb})$ and XV (1091 kb) collectively into 10 minichromosomes ranging in size from 29-631 kb. We also discuss the implications of this technique for studying genome plasticity in $S$. cerevisiae.

\section{MATERIALS AND METHODS}

\section{Strains and Media}

S. cerevisiae strain FY833 (11)

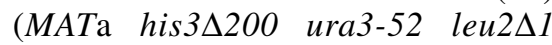
lys $2 \Delta 202 \operatorname{trp} 1 \Delta 63$ ) was used for chromosome-splitting experiments. Yeast cells were grown on YPDA medium containing $1 \%$ yeast extract, $2 \%$ peptone, $2 \%$ glucose, and $0.04 \%$ adenine (Sigma-Aldrich, St. Louis, MO, USA), SD medium containing $2 \%$ glucose and $0.67 \%$ Difco $^{\mathrm{TM}}$ yeast nitrogen base without amino acids (YNB; BD Biosciences, San Jose, CA, USA), supplemented as needed with amino acids and bases to cover auxotrophic requirements or SG medium containing $2 \%$ galactose and $0.67 \%$ YNB supplemented with amino acids and bases as noted above. 


\section{Plasmid Construction}

Plasmids p1417, p1418, and 1419 carrying the Candida glabrata HIS3 (CgHIS3), TRPl (CgTRP1), and LEU2 (CgLEU2) genes, respectively, were used as templates for markergene amplifications (Reference 12; also see www.ncbi.nlm.nih.gov). Plasmids p3008, p3009, p3010, p3121, p3124 contain loxP-CgLEU2-loxP, loxP-CgHIS3-loxP, loxP-CgTRP1loxP, CEN4, CEN4-loxP-CgTRP1loxP cassettes, respectively (see the supplementary material online at www.BioTechniques.com for details). Plasmid pUG6 (13) containing a loxP-KanMX-loxP module was used as a backbone for the construction of plasmids described above. Plasmids p3012 and p3027 carry CEN4 and loxP-CgHIS3-loxP cassettes within the pBluescript ${ }^{\circledR}$ II SK SStratagene, $^{-}$ La Jolla, CA, USA) backbone, respectively. Cre recombinase expression vector pSH47 has been previously described (13).

\section{PCR-Mediated Preparation of Splitting Fragments}

For splitting chromosome I, each of the splitting fragments with short homology extension was produced by PCR with the following PCR conditions: $94^{\circ} \mathrm{C}$ for $5 \mathrm{~min}$, followed by 30 cycles of $94^{\circ} \mathrm{C}$ for $30 \mathrm{~s}, 55^{\circ} \mathrm{C}$ for 30 $\mathrm{s}$, and $72^{\circ} \mathrm{C}$ for $2 \mathrm{~min}$. For splitting chromosome $\mathrm{XV}$, each of the target sequences was initially generated by PCR using similar conditions as described above. Overlap-extension PCR was carried out in a $100-\mu \mathrm{L}$ reaction volume containing $1 \mu \mathrm{M}$ of each primer, $50 \mathrm{ng}$ of each PCR product, and $2.5 \mathrm{U}$ TaKaRa Ex Taq ${ }^{\mathrm{TM}}$ polymerase (Takara Shuzo, Kyoto, Japan). The conditions of the reaction were 1 cycle of $94^{\circ} \mathrm{C}$ for $5 \mathrm{~min}$, and then 30 cycles of $94^{\circ} \mathrm{C}$ for $30 \mathrm{~s}, 55^{\circ} \mathrm{C}$ for $30 \mathrm{~s}$, and $72^{\circ} \mathrm{C}$ for $2.5 \mathrm{~min}$. PCR products were analyzed on $0.7 \%$ agarose gels stained with ethidium bromide. Typically, $5 \mu \mathrm{g}$ DNA were obtained from a single PCR. The PCR products were ethanol-precipitated and subsequently used to transform yeast.

\section{Yeast Transformation}

Yeast transformations were performed according to Gietz and Schiestl (14). Approximately $15 \mu \mathrm{g}$ of each PCR fragment were used to split chromosome I while approximately 5 $\mu \mathrm{g}$ of each PCR fragment were used to split chromosome XV.

\section{Marker Rescue Procedure}

The Cre recombinase expression plasmid pSH47 marked with URA3 was introduced into $\mathrm{Leu}^{+} \mathrm{His}^{+} \mathrm{Trp}^{+}$transformants. Following transformation, the cells were grown on an SD-leu-hisura-trp plate and then shifted to SGura medium to induce the expression of the Cre recombinase. Following overnight growth, the cells were spread on YPDA plates after the appropriate dilution and colonies that formed were replica-plated to SD-leu, SD-his, SDura, and SD-trp plates to check for marker and plasmid loss. Excision of the marker genes and karyotypes of clones that showed leucine, histidine, uracil, and tryptophan auxotrophies were confirmed by pulsed-field gel electrophoresis (PFGE) and Southern blot analysis.

\section{Pulsed-Field Gel Electrophoresis and Southern Blot Analysis}

Chromosomal DNAs of S. cerevisiae embedded in agarose plugs were prepared according to the procedure of Sheehan and Weiss (15). Chromosomes were separated on a $1 \%$ gel by PFGE using the CHEF Mapper ${ }^{\circledR}$ XA pulsed-field electrophoresis system (Bio-Rad Laboratories, Hercules, CA, USA) in $0.5 \times$ TBE (Tris-borateEDTA) buffer at $14^{\circ} \mathrm{C}$ using conditions described in the figure legends. After staining with ethidium bromide, DNA was transferred onto Hybond ${ }^{\mathrm{TM}}-\mathrm{N}^{+}$membranes (Amersham Biosciences, Piscataway, NJ, USA) by capillary blotting, and the membranes were hybridized with probes generated by PCR using specific primer pairs. Probe labeling, hybridization, and hybridization signal detection were carried out using the ECL direct ${ }^{\mathrm{TM}}$ nucleic acid labeling and detection system (Amersham Biosciences).

\section{RESULTS}

A yeast chromosome in haploid cells can be split into two chromosomes by the integration of a chromosome-splitting vector harboring a target sequence, selection marker, CEN4 sequence, and inverted repeat of termini of Tetrahymena ribosomal DNA sequence (Tr) (16) into the target site (Figure 1). To overcome the limitations associated with the current method of chromosome splitting (see Introduction), we have developed a simple but highly efficient method, which we call PCR-mediated chromosome splitting (PCS; Figure 2A). To make these advances, we first adopted the use of six copies of the $5^{\prime}$-CCCCAA- $3^{\prime}$ repeat sequence, $5^{\prime}-\left(\mathrm{C}_{4} \mathrm{~A}_{2}\right)_{6}-3^{\prime}$, as a seed for telomere formation in yeast (17) instead of the Tr used in the previous method (Figure 1), which interfered with PCR ampli-

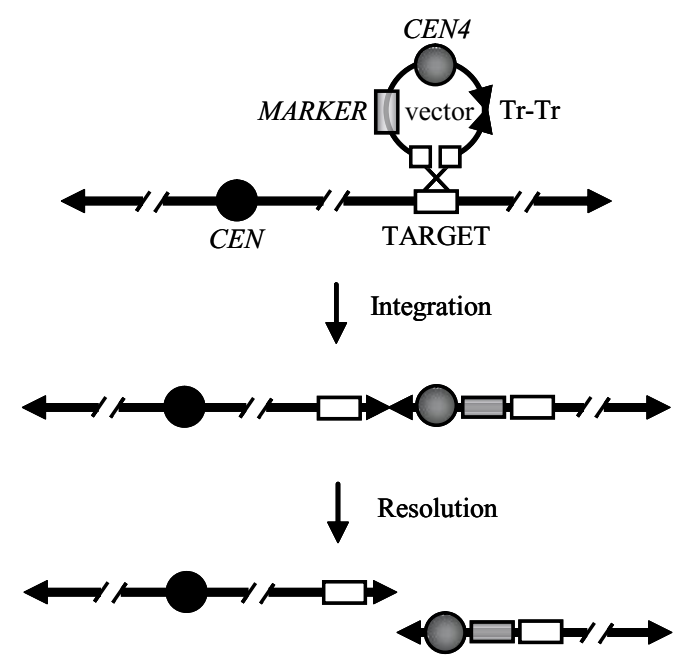

Figure 1. Diagram of chromosome splitting. In the first step, a splitting vector harboring an inverted repeat of termini of Tetrahymena ribosomal DNA sequence (Tr) consisting of about 50 copies of the $5^{\prime}$-CCCCAA- $3^{\prime}$ repeat sequence integrates into the target site. Next, the Tr ends resolve into two new telomeres, resulting in the splitting of the chromosome into two monocentric chromosomes. 
fication in our preliminary study. With this new design, it became possible to prepare marker and CEN4 cassettes for splitting by PCR. Second, a Cre/ loxP system (13) was incorporated to facilitate repeated chromosomesplitting events through the targeted deletion of markers. LEU2 (CgLEU2), HIS3 (CgHIS3), and TRPI (CgTRPl) from C. glabrata $(78 \%, 64 \%$, and $61 \%$ identity, respectively, with the corresponding $S$. cerevisiae genes at nucleotide sequence) were used to avoid undesirable recombination between the splitting fragments and the marker loci within the S. cerevisiae genome.

To test the performance of the PCS method, we initially carried out repeated chromosome splitting with short (45 bp) homologous extensions. In this report, chromosomal segments containing at least one ARS predicted by Wyrick et al. (18) were engineered into split chromosomes. A site approximately $181 \mathrm{~kb}$ from the left end of chromosome I (approximately $230 \mathrm{~kb}$ ) was chosen as the splitting site because breakage here generates an approximately $50-\mathrm{kb}$ minichromosome containing no known essential genes, according to the Saccharomyces Genome Database (SGD ${ }^{\mathrm{TM}}$; www. yeastgenome.org) (Figure 2B). loxPCgHIS3-loxP and CEN4 cassettes flanked by the short homologous region and the $5^{\prime}-\left(\mathrm{C}_{4} \mathrm{~A}_{2}\right)_{6}{ }^{-3^{\prime}}$ repeat sequence were generated by PCR and introduced into haploid strain FY833 (11). Primers used for chromosome splitting are listed in Supplementary Table S1. Of the $58 \mathrm{His}^{+}$transformants obtained, 27 were analyzed for karyotype by PFGE and Southern blot analysis. The results are summarized in Table 1. As shown in Figure 2C, lane 2, the band corresponding to intact chromosome I was not detected while it was present in the parental strain (lane 1). Instead, two new bands corresponding to 183- and $50-\mathrm{kb}$ fragments were observed in one $(1 / 27=4 \%)$ transformant. A hybridization signal generated by a probe for SWH1 on the $50-\mathrm{kb}$ minichromosome confirmed that chromosome I was successfully split (data not shown). Further, probes for $\mathrm{CgHIS} 3$ and CEN4 hybridized as expected with the 183and the 50-kb minichromosomes, respectively (data not shown). The large majority of other transformants displayed a wild-type karyotype (data not shown).

Because of the low efficiency of chromosome splitting, we evaluated the effect of the length of homology on splitting efficiency. To do this, the 183-kb minichromosome generated in our initial experiment was split again into 111- and 74-kb fragments (each containing essential genes) by using primers having 80 nucleotides of homology to the target sequence (Figure 2B). Of $17 \mathrm{Trp}^{+}$transformants obtained, $6(35 \%)$ showed that the 183-kb minichromosome had been successfully split (Figure $2 \mathrm{C}$, lane 3 ), suggesting that the splitting efficiency was substantially enhanced by increasing the length of homologous sequence. For this reason, we used fragments with 80 bp of homology at either end for the subsequent splitting of chromosome I. When the resulting $111-\mathrm{kb}$ minichromosome was further split into 71- and 43-kb fragments (Figure 2C, lane 4), the splitting frequency was found to be $17 \%$. In order to use the markers repeatedly for subsequent splitting, a marker-rescue procedure was used (see Materials and Methods). In our protocol, although approximately $80 \%$ of the clones recovered underwent the correct excision of the three markers, approximately $8 \%$ of the recovered clones showed simultaneous loss of
A

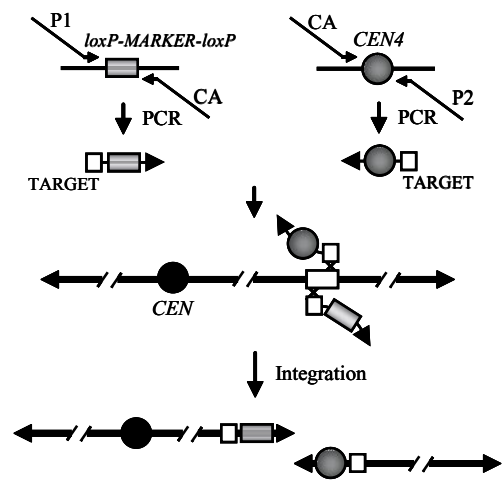

B

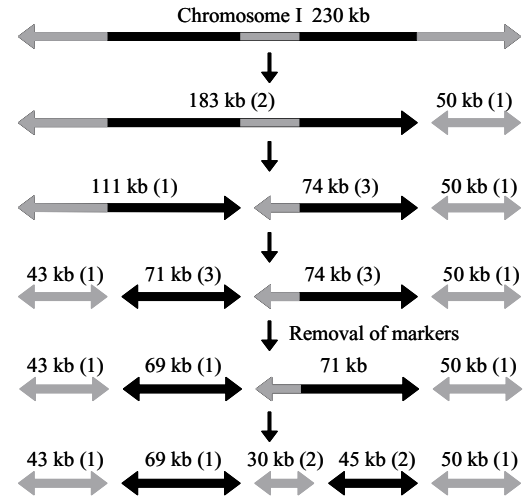

C

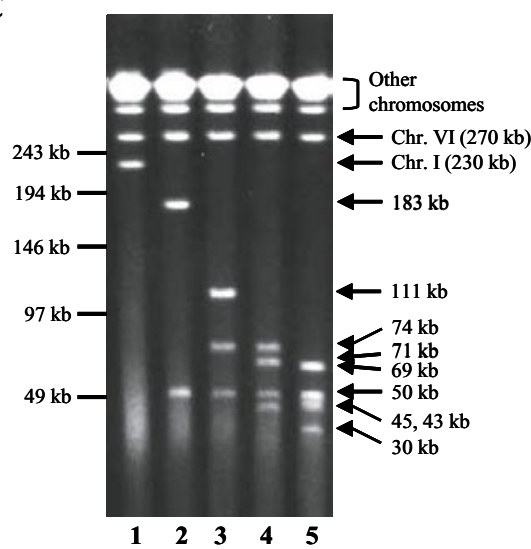

Figure 2. Multiple splitting of chromosome I. (A) The PCS method with short homology extension. The loxP-MARKER-loxP and CEN4 cassettes were generated by PCR using two oligonucleotides: one having a short region of homology ( 45 or 80 bp) to the target site (P1 and P2), and the other having six copies of the $5^{\prime}$-CCCCAA- $3^{\prime}$ repeat sequence, $5^{\prime}-\left(\mathrm{C}_{4} \mathrm{~A}_{2}\right)_{6}-3^{\prime}(\mathrm{CA})$. Simultaneous integration of two PCR fragments results in the splitting of a chromosome at the target site into two monocentric chromosomes. (B) Diagram showing the splitting of chromosome I. Chromosomal regions carrying nonessential genes are indicated in gray. The numbers in parentheses are the increase in length in kilobase pairs of the minichromosomes due to the integrated splitting cassettes, but without regard to changes in size due to the new telomeres. (C) Electrophoretic karyotypes of strains having split chromosomes originating from chromosome I. Run conditions were generated by the autoalgorithm mode of the CHEF Mapper PFGE system by using a size range of 10-250 kb. Numbers in kilobase pairs without a chromosomal designation represent newly generated split chromosomes. DNA samples were prepared from the following strains: the parental strain, FY833 (lane 1); a strain having two split chromosomes originating from chromosome I (lane 2); a strain having three split chromosomes originating from chromosome I (lane 3); a strain having four split chromosomes originating from chromosome I (lane 4); and a strain having five split chromosomes originating from chromosome I (lane 5). The 45- and 43-kb minichromosomes overlap with one another in lane 5. The positions of $\lambda$ ladders (Bio-Rad Laboratories) are indicated on the left-hand side of the panel. PCS, PCR-mediated chromosome splitting; PFGE, pulsed-field gel electrophoresis. 
Table 1. Description of Split Loci and Their Splitting Efficiencies

\begin{tabular}{|c|c|c|c|c|c|}
\hline Locus Split ${ }^{a}$ & Templates $^{b}$ & $\begin{array}{l}\text { Homology } \\
\text { (bp) }\end{array}$ & $\begin{array}{l}\text { Transformants } \\
\text { (n) }\end{array}$ & $\begin{array}{l}\text { Split } \\
\text { Transformants } \\
\text { (\%) }\end{array}$ & $\begin{array}{l}\text { Transformants with Lost } \\
\text { Minichromosomes } \\
\text { (\%) }\end{array}$ \\
\hline Chr. I 181,213.5 & p3027, p3012 & 45 & 58 & $4(1 / 27)$ & $33(9 / 27)$ \\
\hline Chr. I 109,577.5 & p3010, p3121 & 80 & 17 & $35(6 / 17)$ & N.A. \\
\hline Chr. I 42,447.5 & p3008, p3121 & 80 & 173 & $17(5 / 29)$ & $10(3 / 29)$ \\
\hline Chr. I 137,799.5 & p3009, p3124 & 80 & 52 & $17(5 / 29)$ & $0(0 / 29)$ \\
\hline Chr. XV 672,254.5 & p3008, p3121 & approx. 400 & 648 & $86(12 / 14)$ & N.A. \\
\hline Chr. XV 1,044,842.5 & p3010, p3121 & approx. 400 & 1624 & $71(17 / 24)$ & $17(4 / 24)$ \\
\hline Chr. XV 702,299.5 & p3009, p3121 & approx. 500 & 1944 & $89(17 / 19)$ & $0(0 / 19)$ \\
\hline \multicolumn{6}{|c|}{ 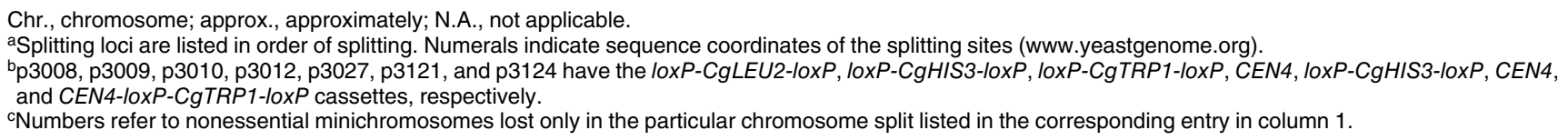 } \\
\hline
\end{tabular}

the markers and plasmid. Although a relatively long-term induction (approximately $12 \mathrm{~h}$ ) of the Cre recombinase was needed for the simultaneous excision of the 3 markers, none of the 12 clones recovered appeared to have undergone chromosomal rearrangements based on karyotype analysis (data not shown). When the 71-kb minichromosome originating from the middle region of chromosome I was split into 45- and 30-kb fragments, the frequency of splitting was again found to be $17 \%$ (Figure 2C, lane 5). Southern blot analyses performed with probes specific for each split chromosome confirmed that splitting occurred as expected (data not shown).

To investigate whether we could further improve the effectiveness of the PCS method, the target sequence was extended to about $400 \mathrm{bp}$ in length using overlap-extension PCR $(19,20)$ (Figure 3A). Using the same strain in
A

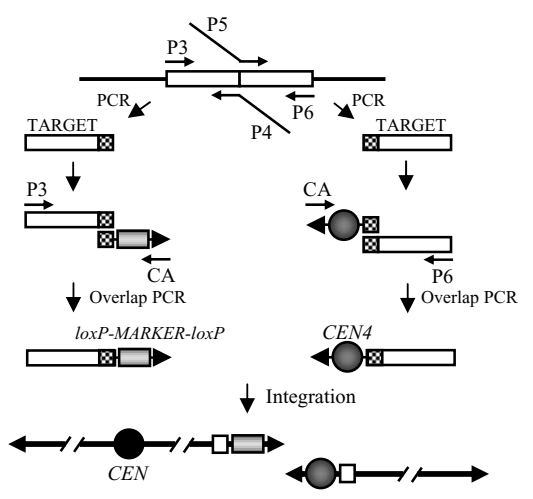

B

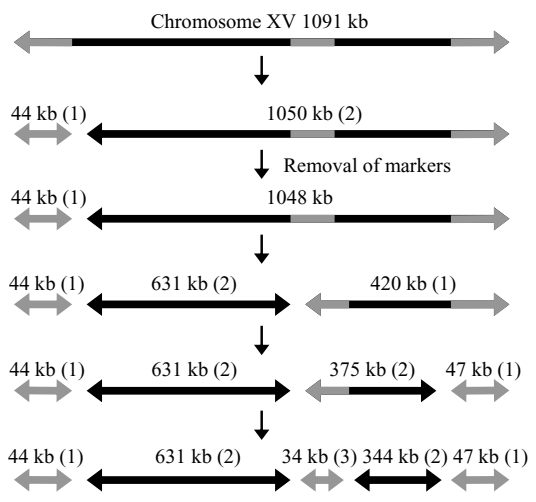

C

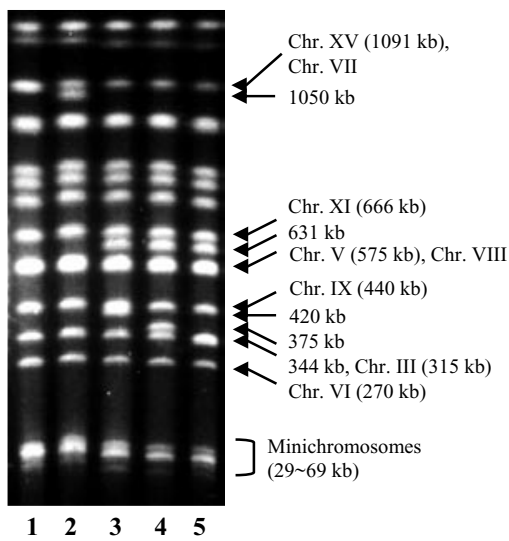

Figure 3. Multiple splitting of chromosome XV. (A) The PCS method with long homology extension. First, two target fragments having an additional 30-bp sequence (hatched box) were amplified by PCR using two sets of oligonucleotides, P3 (20 nucleotides) and P4 (50 nucleotides), and P5 (50 nucleotides) and P6 (20 nucleotides). The resulting PCR products were gel-purified and then mixed with the loxP-MARKER-loxP or CEN4 cassette flanked by the same 30-bp sequence and the $5^{\prime}-\left(\mathrm{C}_{4} \mathrm{~A}_{2}\right)_{6}-3^{\prime}$ sequence. These marker and $C E N 4$ cassettes were prepared by PCR using primers loxP-cas (see Supplementary Table S1) and $\mathrm{CA}$, pooled in advance as stock solutions, and were used to generate splitting fragments with long homology extensions in the second PCR using primers P3 and CA, and P6 and CA, respectively. (B) Diagram showing the splitting of chromosome XV. Chromosomal regions carrying nonessential genes are indicated in gray. Numbers in parentheses are the increase in length in kilobase pairs of the minichromosomes due to the integrated splitting cassettes, but without regard to the change in size due to the new telomeres. (C) Electrophoretic karyotypes of strains having split chromosomes originating from chromosomes I and XV. Chromosomes were separated with a 60 -s pulse for $15 \mathrm{~h}$, followed by a 90 -s pulse for $9 \mathrm{~h}$, at $6 \mathrm{~V} / \mathrm{cm}$. Numbers in kilobase pairs without a chromosomal designation represent newly generated split chromosomes. DNA samples were prepared from the following strains: a strain having intact chromosome XV (lane 1); a strain having two split chromosomes originating from chromosome XV (lane 2); a strain having three split chromosomes originating from chromosome $\mathrm{XV}$ (lane 3); a strain having four split chromosomes originating from chromosome XV (lane 4); and a strain having five split chromosomes originating from chromosome XV (lane 5). In lane 2, the 44-kb fragment overlaps with some of the minichromosomes derived from chromosome I under these electrophoretic conditions. Similarly, in lane 4, a 47-kb fragment overlaps with several minichromosomes. In lane 5, the 344- and 34-kb fragments overlap with chromosome III (315 kb) and with some minichromosomes, respectively. PCS, PCR-mediated chromosome splitting; , approximately. 
which we had already split chromosome I into five fragments, we attempted to split chromosome XV (approximately $1091 \mathrm{~kb}$ ) into five minichromosomes with the overlap-extension PCR (Figure 3B). First, to split chromosome XV into 1050- and 44-kb minichromosomes, two splitting fragments containing long homologous extensions were prepared as described in the Materials and Methods section and used for transformation. Transformation with $5 \mu \mathrm{g}$ of each PCR fragment yielded approximately $1500 \mathrm{Leu}^{+}$transformants (Table 1). Among 10 transformants analyzed, although one transformant had an intact chromosome XV, 7 (70\%) showed that successful splitting occurred at the correct genomic locus (Figure 3C, lane 2). We noted that the number of transformants increased significantly relative to our previous PCS experiments using shorter (45 or $80 \mathrm{bp}$ ) regions of homology and that the efficiency of splitting also improved drastically. With this alteration in the protocol, a similarly high splitting frequency was obtained in all the subsequent experiments. After excision of the markers, we next split the resulting 1048-kb minichromosome into 631and $420-\mathrm{kb}$ fragments (Figure $3 \mathrm{C}$, lane 3 ) at an efficiency of $86 \%$. Similarly, the resulting $420-\mathrm{kb}$ minichromosome was subsequently split into 375 - and the 47-kb fragments with an efficiency of $71 \%$ (Figure 3C, lane 4). Finally, the resulting $375-\mathrm{kb}$ minichromosome was split into 344- and the 34-kb fragments with an efficiency of $89 \%$ (Figure 3C, lane 5). Southern blot analyses performed with probes specific for each minichromosome confirmed that chromosome $\mathrm{XV}$ was successfully split into five fragments that were maintained in the respective clones (data not shown). These results demonstrate that the PCS method developed in this study is highly efficient and provides a powerful tool for dissecting the yeast genome.

\section{DISCUSSION}

We have described a novel genomeengineering technique called the PCS method for splitting yeast chromosomes efficiently. Two main features of this method are (i) the incorporation of a two-step PCR with overlap extension for preparing the splitting fragments and (ii) the Cre-loxP system for eliminating the markers used. This protocol makes it possible to split chromosomes simply, repeatedly, and with high efficiency (approximately $80 \%$ ). Because physical dissection of a chromosome provides a useful resource (i.e., a set of derived minichromosomes with various components, lengths, and stabilities), the PCS method should contribute to our understanding of genome organization in yeast and, by extension, in other eukaryotes. This method may also lead to the improvement of industrially important strains of yeast through the introduction of specific chromosomal segments.

We found that transformants that had lost a newly generated minichromosome carrying nonessential genes appeared in some transformation steps at frequencies of $10 \%$ to $33 \%$ (Table 1). Minichromosome loss could occur either as a consequence of incomplete splitting (such as the integration of one of two of the splitting fragments into the target) or because of spontaneous loss during subsequent cultivation. Because of the limited cultivation time following transformation, it is expected that these transformants result from incomplete splitting. However, we cannot rule out the possibility that these transformants result from postsplitting loss of the minichromosome because these same minichromosomes can be lost with high frequency during the growth of the transformant. In fact, during the course of the current study, we found that most split chromosomes carrying nonessential genes could be lost in various combinations during cultivation in rich or minimal media. These minichromosomes (i.e., the 50-, 43-, 44-, and 47-kb fragments) carry $23,21,19$, and 21 open reading frames (ORFs), respectively (Figures $2 \mathrm{~B}$ and $3 \mathrm{~B}$ ). The clone with the smallest genome obtained in this study had lost the 50-, 44-, and 47-kb fragments (138 $\mathrm{kb}$ in total chromosomal segments), indicating that synthetic lethal interactions did not occur among the 63 ORFs contained therein. In contrast, while minichromosomes of less than $100 \mathrm{~kb}$ in length have been reported to display dramatically decreased mitotic stability (21-23), we did not detect loss of the 30- or 34-kb minichromosomes, which apparently did not contain essential genes, suggesting that these chromosomal segments were important for survival. Therefore, the PCS method provides a convenient tool to identify nonessential gene clusters. In the future, the PCS method may offer a practical approach to identify minimal genome compositions for $S$. cerevisiae, based on the principle that various combinations of nonessential chromosome fragments can be lost as a function of growth conditions. Yeast cells with a variety of unique genome constitutions created by combinatorial loss of minichromosomes (e.g., more than $10^{9}$ combinations for 30 minichromosomes) will be a valuable resource to better understand genome plasticity of free-living eukaryotic cells.

\section{ACKNOWLEDGMENTS}

We thank Fred Winston for the gift of the FY833 strain; Johannes H. Hegemann for providing plasmids pUG6 and pSH47; and Kunio Kitada for sending plasmids p1417, p1418, and p1419. This study was partially supported by the Ministry of Education, Culture, Sports, Science and Technology, a grant-in-aid for Scientific Research B, 13580064, 2003-2005 (to S.H.), and was carried out as a part of The Project for Development of a Technological Infrastructure for Industrial Bioprocesses on $R \& D$ of New Industrial Science and Technology Frontiers by the Ministry of Economy, Trade \& Industry (METI), and entrusted by the New Energy and Industrial Technology Development Organization (NEDO) (to S.H.).

\section{COMPETING INTERESTS STATEMENT}

The authors declare no conflicts of interest.

\section{REFERENCES}

1.Kolisnychenko, V., G. Plunkett III, C.D. Herring, T. Fehér, J. Pósfai, F.R. Blattner, and G. Pósfai. 2002. Engineering a reduced Escherichia coli genome. Genome Res. 
12:640-647.

2.Yu, B.J., B.H. Sung, M.D. Koob, C.H. Lee, J.H. Lee, W.S. Lee, M.S. Kim, and S.C. Kim. 2002. Minimization of the Escherichia coli genome using a Tn5-targeted Cre/loxP excision system. Nat. Biotechnol. 20:10181023.

3.Delneri, D., I. Colson, S. Grammenoudi, I.N. Roberts, E.J. Louis, and S.G. Oliver. 2003. Engineering evolution to study speciation in yeasts. Nature 422:68-72.

4.Yang, J.W., C. Pendon, J. Yang, N. Haywood, A. Chand, and W.R. Brown. 2000. Human mini-chromosomes with minimal centromeres. Hum. Mol. Genet. 9:1891-1902.

5.Westers, H., R. Dorenbos, J.M. van Dijl, J. Kabel, T. Flanagan, K.M. Devine, F. Jude, S.J. Seror, et al. 2003. Genome engineering reveals large dispensable regions in Bacillus subtilis. Mol. Biol. Evol. 20:2076-2090.

6.Widianto, D., Y. Mukai, K.-H. Kim, S. Harashima, and Y. Oshima. 1996. One-step splitting of a chromosome in haploid cells of Saccharomyces cerevisiae and its effect on the cell proliferation. J. Ferment. Bioeng. 82:199204

7.Widianto, D., E. Yamamoto, M. Sugiyama, Y. Mukai, Y. Kaneko, Y. Oshima, M. Nishizawa, and S. Harashima. 2003. Creating a Saccharomyces cerevisiae haploid strain having 21 chromosomes. J. Biosci. Bioeng. 95:89-94.

8.Vollrath, D., R.W. Davis, C. Connelly, and P. Hieter. 1988. Physical mapping of large DNA by chromosome fragmentation. Proc. Natl. Acad. Sci. USA 85:6027-6031.

9.Widianto, D., E. Yamamoto, Y. Mukai, Y. Oshima, and S. Harashima. 1997. A method for fusing chromosomes in Saccharomyces cerevisiae. J. Ferment. Bioeng. 83:125-131.

10.Mizukami, A., E. Nagamori, Y. Takakura, S. Matsunaga, Y. Kaneko, S. Kajiyama, S. Harashima, A. Kobayashi, et al. 2003. Transformation of yeast using calcium alginate microbeads with surface-immobilized chromosomal DNA. BioTechniques 35:738740 .

11.Winston, F., C. Dollard, and S.L. RicuperoHovasse. 1995. Construction of a set of convenient Saccharomyces cerevisiae strains that are isogenic to $\mathrm{S} 288 \mathrm{C}$. Yeast 11:53-55.

12.Kitada, K., E. Yamaguchi, and M. Arisawa. 1995. Cloning of the Candida glabrata TRP1 and HIS3 genes, and construction of their disruptant strains by sequential integrative transformation. Gene 165:203-206.

13.Güldener, U., S. Heck, T. Fiedler, J. Beinhauer, and J.H. Hegemann. 1996. A new efficient gene disruption cassette for repeated use in budding yeast. Nucleic Acids Res. 24:2519-2524.

14.Gietz, R.D. and R.H. Schiestl. 1995. Transforming yeast with DNA. Methods Mol. Cell. Biol. 5:255-269.

15.Sheehan, C. and A.S. Weiss. 1990. Yeast artificial chromosomes: rapid extraction for high resolution analysis. Nucleic Acids Res. 18:2193.

16.Szostak, J.W. and E.H. Blackburn. 1982. Cloning yeast telomeres on linear plasmid vectors. Cell 29:245-255.

17.Murray, A.W., T.B. Claus, and J.W.Szostak.
1988. Characterization of two telomeric DNA processing reactions in Saccharomyces cerevisiae. Mol. Cell. Biol. 8:4642-4650.

18.Wyrick, J.J., J.G. Aparicio, T. Chen, J.D. Barnett, E.G. Jennings, R.A. Young, S.P. Bell, and O.M. Aparicio. 2001. Genomewide distribution of ORC and MCM proteins in $S$. cerevisiae: high-resolution mapping of replication origins. Science 294:2357-2360.

19.Ho, S.N., H.D. Hunt, R.M. Horton, J.K. Pullen, and L.R. Pease. 1989. Site-directed mutagenesis by overlap extension using the polymerase chain reaction. Gene 77:51-59.

20.Dillon, P.J. and C.A. Rosen. 1990. A rapid method for the construction of synthetic genes using the polymerase chain reaction. BioTechniques 9:298-299.

21.Hieter, P., C. Mann, M. Snyder, and R.W. Davis. 1985. Mitotic stability of yeast chromosomes: a colony color assay that measures nondisjunction and chromosome loss. Cell 40:381-392.

22.Surosky, R.T., C.S. Newlon, and B.-K. Tye. 1986. The mitotic stability of deletion derivatives of chromosome III in yeast. Proc. Natl. Acad. Sci. USA 83:414-418.

23.Murray, A.W., N.P. Schultes, and J.W. Szostak. 1986. Chromosome length controls mitotic chromosome segregation in yeast. Cell 45:529-536.

Received 13 October 2004; accepted 7 January 2005.

Address correspondence to:

Satoshi Harashima

Department of Biotechnology

Graduate School of Engineering,

Osaka University

2-1 Yamadaoka, Suita-shi

Osaka 565-0871, Japan

e-mail: harashima@bio.eng.osaka-u.ac.jp 